\title{
Chaos and hyperchaos in a symmetric coupling of three quadratic maps
}

\author{
João C. Xavier and Paulo C. Rech \\ Manuscript received on August 30, 2009 / accepted on January 25, 2010
}

\begin{abstract}
In this paper we investigate the dynamical behavior of a symmetric coupling of three quadratic maps, and identify various interesting features as a function of the nonlinearity and the coupling parameters. In particular, we study the emergence of quasiperiodic states arising from Naimark-Sacker bifurcations of stable periodic orbits pertaining to $1 \times 2^{n}$ cascade, namely period- 1 and period-2 orbits. Lyapunov exponent plots, parameter-space and three-dimensional phase-space portraits, and bifurcation diagrams are used to study the transition from periodic to quasiperiodic states, from quasiperiodic to mode-locked and to chaotic states, and from chaotic to hyperchaotic states. We also investigate the modifications introduced in the parameter-space, by the increase of the number of maps in the coupling.
\end{abstract}

Keywords: Naimark-Sacker bifurcation, Lyapunov exponents, hyperchaos. 


\section{INTRODUCTION}

The notion of hyperchaos was introduced by Rössler [1], and considers the possibility of more than one direction of mean exponential divergence for attractors in dynamical systems, that is, more than one positive Lyapunov exponent. For continuoustime systems, hyperchaos exists only in higher than or equal to four-dimensional autonomous systems, while in discrete-time systems only in higher than or equal to two-dimensional maps. Generally speaking, hyperchaos is more disordered than chaos and, consequently, hyperchaotic systems have more complicated topological structures and dynamics than chaotic systems. In recent years, hyperchaos has attracted considerable attention because of its theoretical and practical applications in various fields. In secure communications, for example, a hyperchaotic signal can be used to mask a message to be transmitted [2, 3]. In semiconductor lasers subject to optical or electrooptical feedback, the characterization of the hyperchaotic dynamics is relevant for secure optical communication based on chaos encryption [4]. A nonlinear circuit based on impulsive switching can be constructed to obtain a hyperchaotic attractor in the laboratory [5]. A two-dimensional non-invertible return map is derived for this experimental setup, being hyperchaos generation guaranteed by two positive Lyapunov exponents. Hyperchaos also can be find in systems of ordinary differential equations which model chemical reactions chains [6]. In this case, the number of positive Lyapunov exponents is a function of the finite dimension of the system. Control theory can be applied to stabilize hyperchaos. For example, a hyperchaotic Lorenz system constructed via state feedback control can be stabilized to unstable equilibrium, periodic orbits, and quasiperiodic orbits [7]. Furthermore, hyperchaotic systems synchronize. This occurs not only between two identical hyperchaotic systems, but also between two different hyperchaotic systems $[8,9,10,11]$.

The purpose of this paper is to determine numerically the emergence of periodic, quasiperiodic, mode-locked, chaotic, and hyperchaotic states in a symmetric linear coupling of three quadratic maps. As we will see, this model presents a very complex dynamics in the parameter-space. Numerical results including Lyapunov exponents, phase-space plots, and bifurcation diagrams will be used to characterize the various transitions observed.

\section{RESULTS AND DISCUSSION}

Here we consider a discrete-time system, namely, a symmetric linear coupling of three quadratic maps given by:

$$
\begin{aligned}
& x_{t+1}=a-x_{t}^{2}+b y_{t}, \\
& y_{t+1}=a-y_{t}^{2}+b z_{t}, \\
& z_{t+1}=a-z_{t}^{2}+b x_{t},
\end{aligned}
$$

where $x_{t}, y_{t}, z_{t}$ represent dynamical variables, $a$ is the nonlinearity parameter, $b$ is the coupling parameter, and $t=$ $0,1,2, \ldots$ is the discrete time. The parameter-space of the system (2.1) is shown in Fig. 1. It is an isoperiodic diagram obtained by discretizing the parameter interval in a grid of $350 \times$ 350 points equally spaced. For each point $(b, a)$, an orbit of initial condition $\left(x_{0}, y_{0}, z_{0}\right)=(0.01,0.005,0.02)$ converges or to a hyperchaotic, or to a chaotic, or to a quasiperiodic, or to a periodic attractor, or to an attractor at infinity (unbounded attractor), after a transient of $5 \times 10^{4}$ iterates. If the trajectory initialized in $\left(x_{0}, y_{0}, z_{0}\right)$ converges to infinity, that is, diverges, we try until 500 new initializations, always increasing of $1 / 500$ each one of the variables. In Fig. 1 the unbounded attractor (blank region) is indicated by $\infty$, and the bigger regions of different periodicities up to period-50, are identified by integer numbers, which denote the period of the respective region. One sees that the period-doubling bifurcation cascade $1 \times 2^{n}$, also present in the quadratic map, is preserved by the coupling. Additionally, there are three disconnected regions in grey color, indicated by the letter $\mathbf{Q}$, where the motion is quasiperiodic. These quasiperiodic motions are born exactly on the boundary with period-1, period2, and period-4 regions, as a result of Naimark-Sacker (NS) bifurcations $[12,13]$. Also are present chaotic and hyperchaotic regions, indicated by the letters $\mathbf{C}$ and $\mathbf{H}$, respectively. In the remainder of this paper, we are concerned with a detailed numerical investigation of the rich dynamics observed in Fig. 1.

In two plots of Fig. 2 one sees magnifications of the two regions inside the boxes $\mathbf{I}$ and $\mathbf{I I}$ in Fig. 1, being the first region I0cated in the range $-0.2 \leq b \leq 0.1(1.30 \leq a \leq 1.56)$, and the second in the range $0.25 \leq b \leq 0.50(0.4 \leq a \leq 0.9)$. Period-4 and period-8 regions, better seen in Fig. 2(a), have well defined boundaries. For parameter values taken along these boundaries, NS bifurcations occur. This means that inside these two grey regions indicated by $\mathbf{Q}$, we have quasiperiodic motion with four and eight closed curves in phase-space, originated from a period-4 and a period-8 orbit, respectively. Quasiperiodic motion region also can be better seen in Fig. 2(b), now with only one closed curve, originated from a NS bifurcation of a period-1 orbit, while in Fig. 1, we have a large region where happens quasiperiodic motion with two closed curves, this time originated from a period-2 orbit. If more and more amplifications are made in 


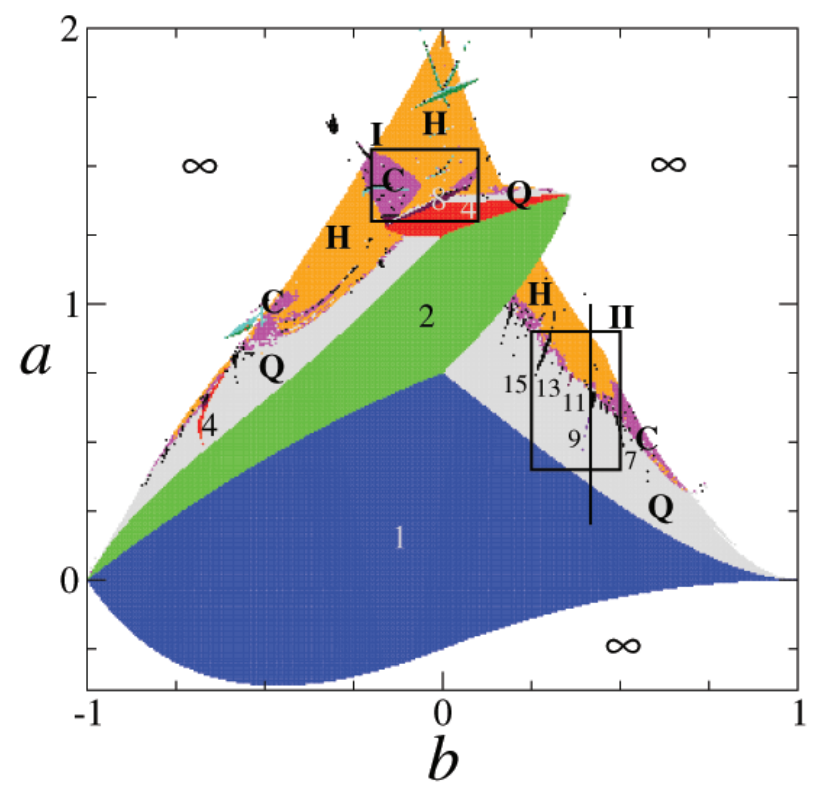

Figure 1 - The parameter-space of the map (2.1). In this and further similar figures, domains of different attractors are identified by different shadings. Numbers indicate periods and letters indicate chaos $(\mathbf{C})$, hyperchaos $(\mathbf{H})$, and quasiperiodicity $(\mathbf{Q})$.
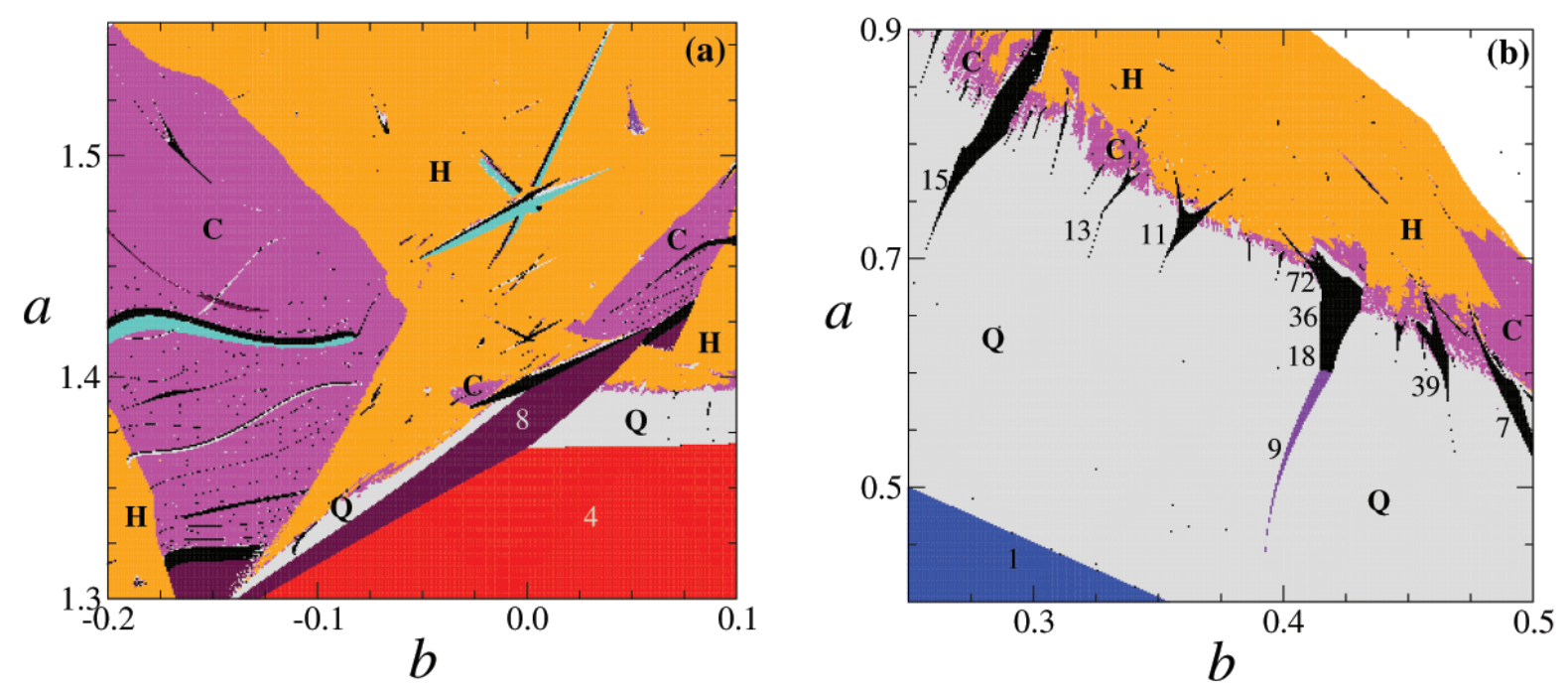

Figure 2 - (a) Magnification of the box I and, (b) magnification of the box II in Fig. 1.

the region inside the box $\mathbf{I}$, more and more quasiperiodic regions will appear, not shown in the scale of Fig. 1, characterized by sixteen, thirty-two, and so on closed curves in phase-space. From Fig. 1 and its amplifications we recognize, immersed in quasiperiodic regions, periodic structures similar to the Arnold tongues observed in the circle map [14].

Figure 3 shows the bifurcation diagram ( $x$ variable), and the largest (continuous line) and the second largest (dashed line) Lyapunov exponent $(\lambda)$ plots for $b=0.4165$ (see black line in Fig. 1) and $0.576<a<0.730$. For the construction of the bifurcation diagram, $10^{3}$ values of the parameter $a$ were considered, and plotted 50 points for each one of them, after a transient of $5 \times 10^{4}$ iterates, always from the initial condition $\left(x_{0}, y_{0}, z_{0}\right)$. Same initial condition, transient, and discretization 
in $a$ axis were utilized for the construction of the largest and the second largest Lyapunov exponent plots, being each $\lambda$ value obtained by averaging over $10^{6}$ iterations.
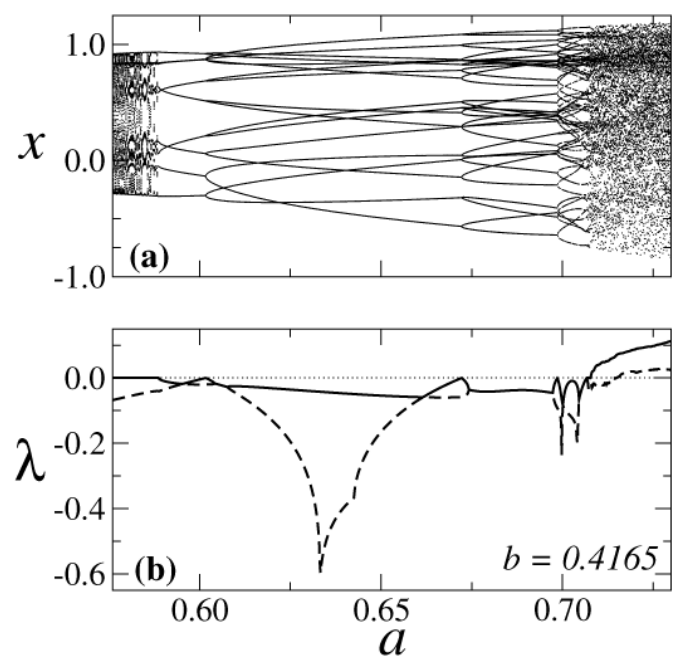

Figure 3 - (a) Bifurcation diagram showing the variable $x$. (b) The largest (continuous line) and the second largest (dashed line) Lyapunov exponent plots. Both graphs as a function of the parameter $a$, for $b=0.4165$.

By decreasing $a$ from 0.576 along the line $b=0.4165$ in Fig. 1, the system is quasiperiodic, characterized by a largest Lyapunov exponent equal to zero, until $a=0.3424$ where period-1 region is reached. This quasiperiodic motion is, therefore, originated from a period-1 orbit, result of a NS bifurcation that occurs at $a=0.3424$. As a consequence of this bifurcation, one invariant closed curve is created, which can be seen in Fig. 4(a), for $a=0.57$. There are mode-locked states (Arnold tongues) immersed in the quasiperiodic region, for which the largest Lyapunov exponent is lesser than zero. By increasing $a$ from 0.576 we find a tongue with period-9, followed by three adjacent domains with period-18, period-36, and period-72 (see Fig. 2(b)). After this finite bifurcation cascade, a NS bifurcation of a period-72 orbit occurs. As a consequence, a new quasiperiodic region is reached, now characterized by seventy-two invariant closed curves, as one sees in Fig. 4(b), for $a=0.707$. Increasing $a$ more, these curves are deformed and finally, chaotic and hyperchaotic states are created at $a=0.708$ and $a=0.715$, respectively. For the chaotic state the largest Lyapunov exponent is greater than zero, while for the hyperchaotic state both, the largest and the second largest Lyapunov exponents, are greater than zero. Chaotic and hyperchaotic attractors appear in Figs. 4(c) and 4 (d), respectively for $a=0.71$ and $a=0.73$. For the chaotic attractor the greater and the second greater Lyapunov exponents are 0.04 and -0.02 , while for the hyperchaotic attractor the correspondent values are 0.11 and 0.02 . The fractal dimension of both attractors was estimated by using the formula of Kaplan and Yorke [14], with the results 2.044 and 2.330, respectively.

Next we use trajectories in the phase-space to observe the dynamics near the boundary with period-1 region in Fig. 1, along the same line $b=0.4165$. Figure 5(a) shows two twodimensional projections of attractors in the phase-space, for different values of parameter $a$, while keeping $b=0.4165$. For $a=0.342$, which is close to the boundary between period-1 and quasiperiodic region, but just before the NS bifurcation, a fixed point is observed. For $a=0.343$, also close the same boundary, but now just after the NS bifurcation, an invariant closed curve (a limit cycle) is observed. Therefore, as the parameter $a$ increases and crosses the NS bifurcation line that separates blue and grey regions in Fig. 1, a fixed point solution is transformed in a quasiperiodic solution characterized by a limit cycle. In Fig. 5(b) is shown the radius of this limit cycle as a function of the difference between the parameter $a$ and its bifurcation value ( $a=0.3424$ ), also for $b=0.4165$. Indeed, numerically we calculate the radius of a limit cycle as an average radius over all points of the trajectory, since the limit cycle is not a circle, mainly far away from the NS bifurcation, where the limit cycle is deformed. In the interval $0<(a-0.3424)<0.25$ the radius of the limit cycle is well approximated by $r=1.69(a-0.3424)^{0.56}$. Note that the exponent value 0.56 is in good agreement with the exact value 0.5 due to the normal form for a NS bifurcation [12].

Up to now we have considered the dynamics of a simple system with only three coupled quadratic maps given by Eqs. (2.1). We have seen that, in spite of its apparent simplicity, our system composed by three coupled quadratic maps displays a much richer bifurcational structure than the period-doubling of its individual components. In sequence we are interested in to observe possible relevant modifications in the parameter-space of the coupling, when the dimensionality of the system grows. Our aim is to check if similar results for parameter-space are obtained to much general systems, i.e., to an array of symmetrically coupled quadratic maps.

The four panels in Fig. 6 refer to five, ten, fifteen, and twenty symmetrically coupled quadratic maps as in system (2.1), respectively. The color code is the same as in Fig. 1, except by the fact that now we prefer to paint the periodic region defined by the period-doubling cascade $1 \times 2^{n}$ with only one color (blue), without separate in different colors the several periods 1, 2, 4, and so on. This region is indicated by the letter $\mathbf{P}$. It can be observed in Figs. $6(\mathbf{a})-6(\mathbf{d})$ that the chaotic region is practically suppres- 

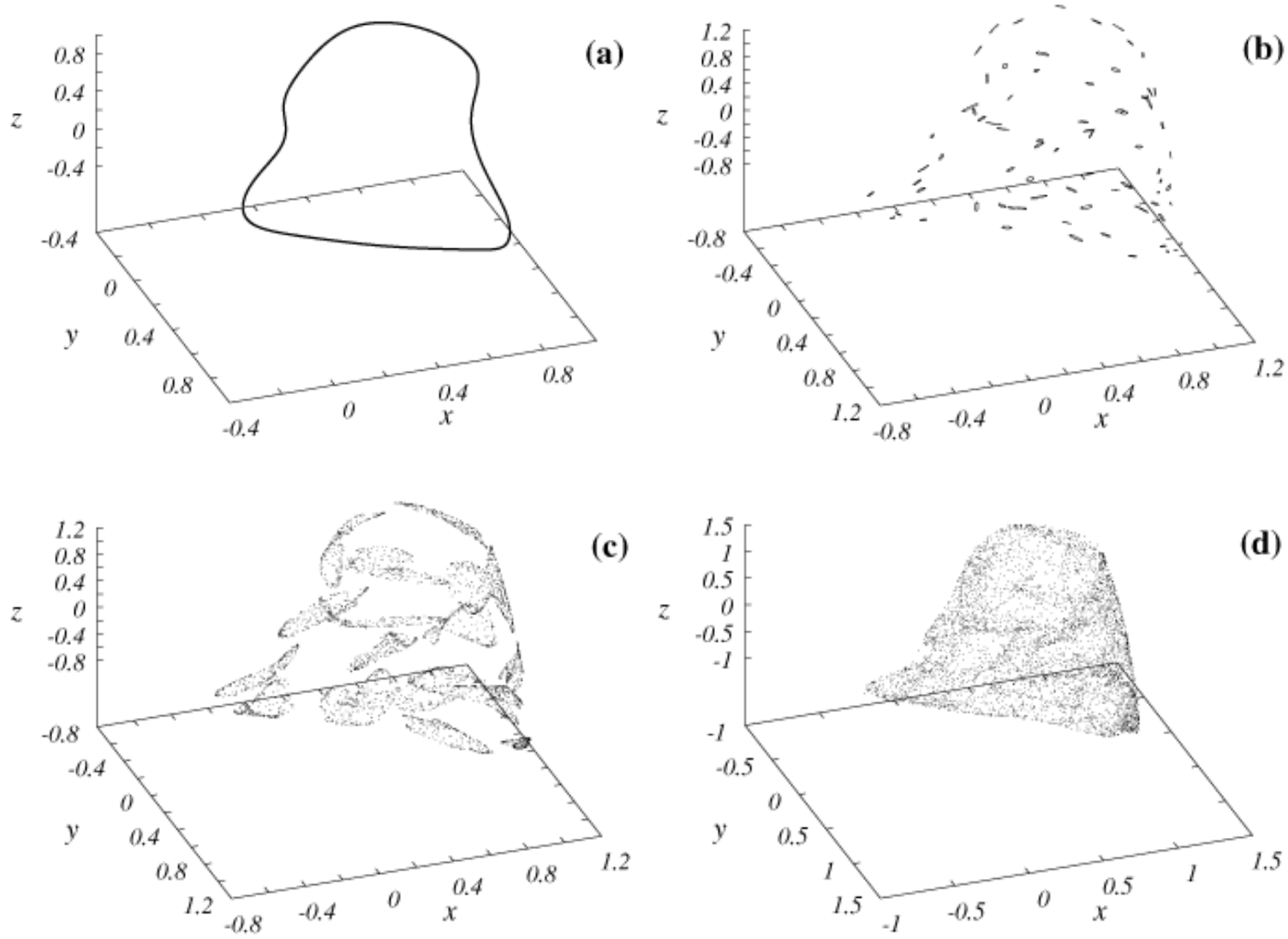

Figure 4 - Four trajectories in the three-dimensional phase-space. (a) Quasiperiodicity, for $a=0.57$. (b) Quasiperiodicity, for $a=0.707$. (c) Chaos, for $a=0.71$. (d) Hyperchaos, for $a=0.73$. Same initial conditions and transient of the others figures were here utilized, being plotted $5 \times 10^{3}$ points.
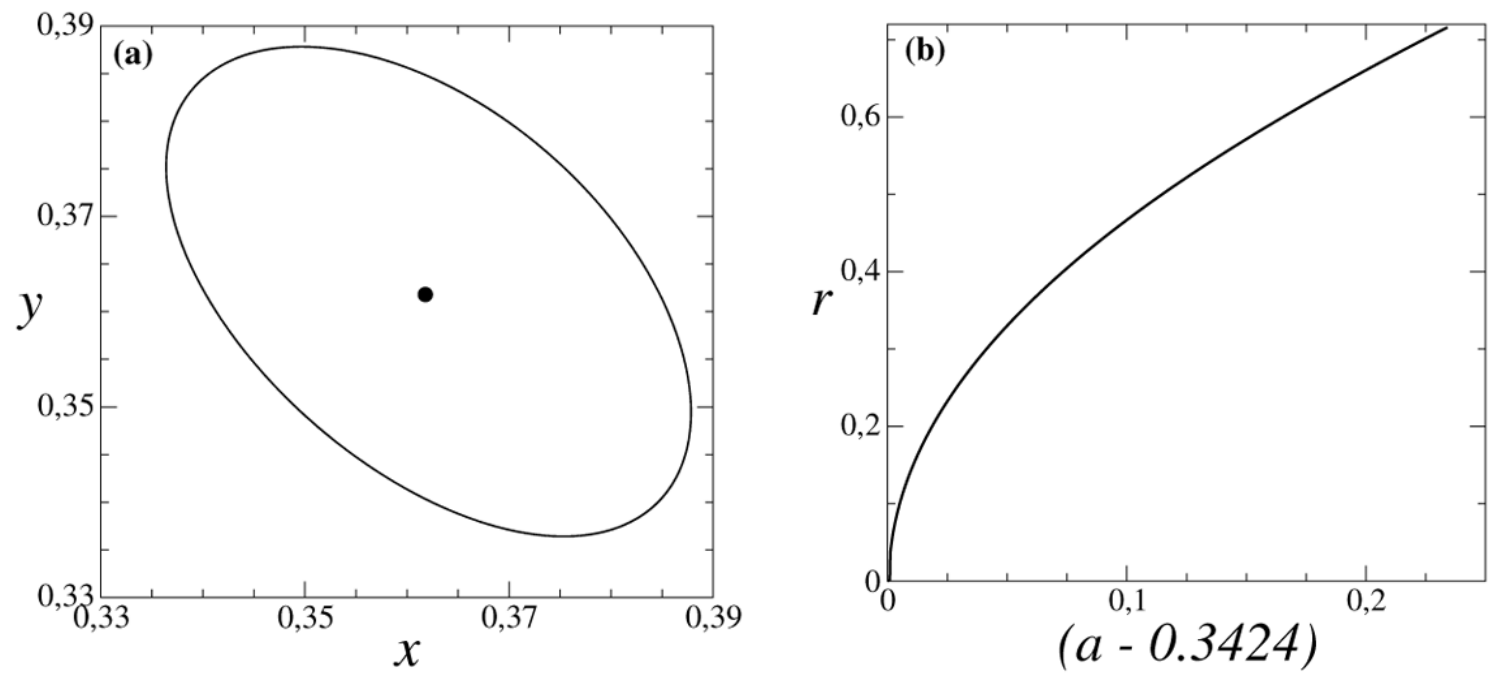

Figure 5 - (a) Two trajectories in the two-dimensional phase-space ( $x y$ plane), showing a fixed point for $a=0.342$, and a limit cycle for $a=0.343$. (b) Radius of the limit cycle as a function of the difference between the parameter $a$ and its bifurcation value 0.3424 . 

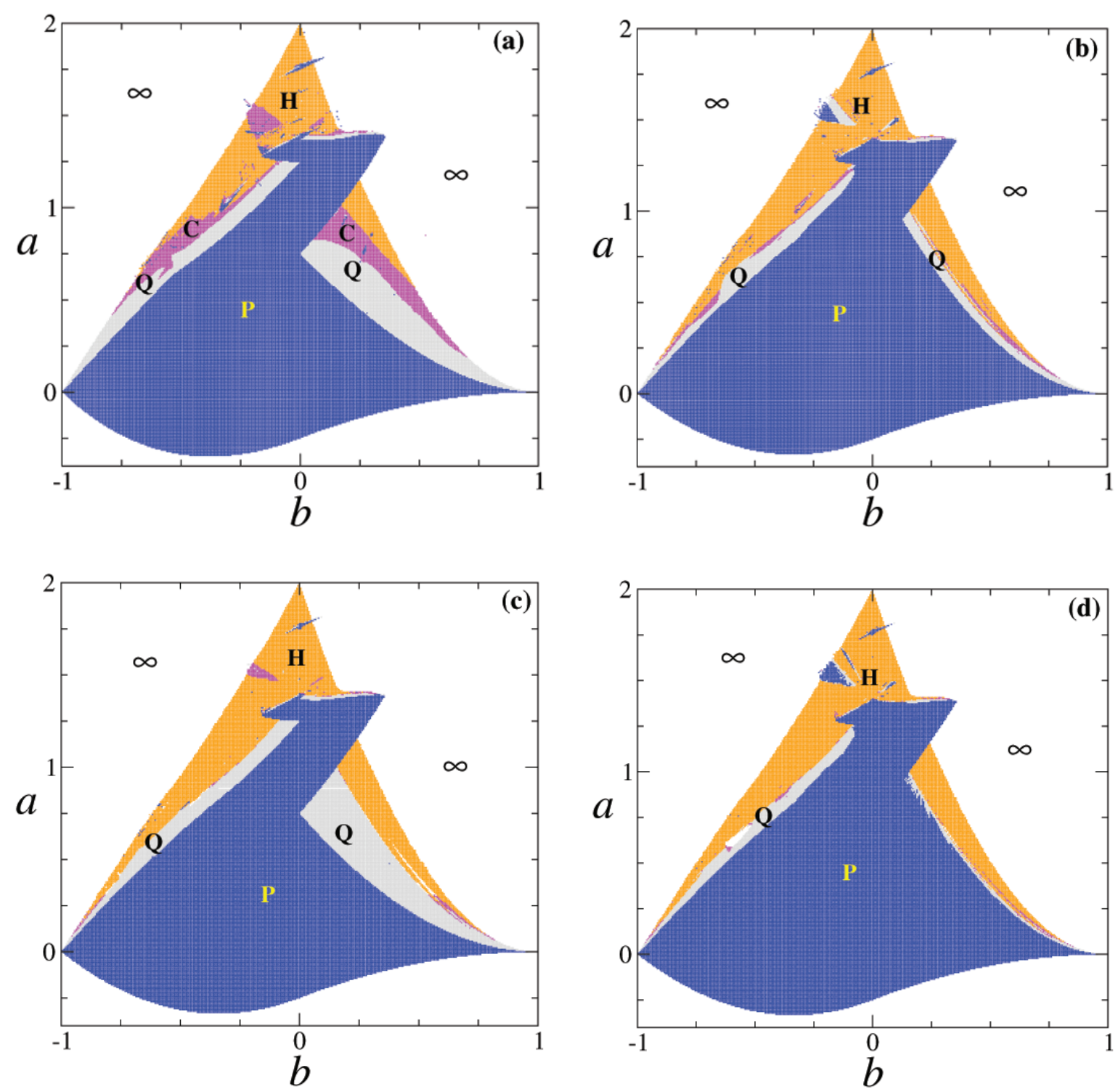

Figure 6 - (a) Five coupled maps. (b) Ten coupled maps. (c) Fifteen coupled maps. (d) Twenty coupled maps. The letter $\mathbf{P}$ indicates periodic region.

sed, and the quasiperiodic region is substantially reduced, as the system dimension grows. Otherwise, periodic and hyperchaotic regions are increased, at the same time. To explain the enlargement of the hyperchaotic region, we conjecture that by growing the system dimension, grows the possibility of the system to present more than one positive Lyapunov exponent, which is characteristic of hyperchaotic systems. We do not have explanation to the enlargement of the periodic region in damage of the shrinkage of the quasiperiodic region. What kind of dynamical phenomena are involved in this Naimark-Sacker bifurcation translation in the parameter-space is something that deserves investigation.

\section{SUMMARY}

In this paper we have considered a three-dimensional discretetime dynamical system with a quadratic nonlinearity, which consists in a symmetric coupling of three quadratic maps (see Eqs. (2.1)). We have shown that this system may exhibits several routes to chaos via quasiperiodicity (see Fig. 2(a)) on account of Naimark-Sacker bifurcations of stable periodic orbits of the $1 \times 2^{n}$ cascade, in addition to the period-doubling route (see Fig. 1) typical of the quadratic map. We also detect a NaimarkSacker bifurcation of a period-72 orbit (see Fig. 4(b)), despite the 
fact that high period stable orbits generally have basins of attraction too small to be detected. We also have shown that, by increasing the number of maps, periodic and hyperchaotic regions are enlarged in parameter-space, while chaotic and quasiperiodic regions are reduced. Numerical results, including Lyapunov exponent plots, parameter-space and phase-space portraits, and bifurcation diagrams were used to describe the transitions from periodic into quasiperiodic states, from quasiperiodic into modelocked or chaotic states, and from chaotic into hyperchaotic states.

\section{ACKNOWLEDGMENTS}

The authors thank Conselho Nacional de Desenvolvimento Científico e Tecnológico - CNPq and Coordenação de Aperfeiçoamento de Pessoal de Nível Superior - CAPES, Brazil, for financial support.

\section{REFERENCES}

[1] RÖSSLER OE. 1979. An equation for hyperchaos. Phys. Lett. A, 71: 155-157.

[2] LI C, LIAO X \& WONG K. 2005. Lag synchronization of hyperchaos with application to secure communications. Chaos Solitons Fractals, 23: 183-193.

[3] QI G, VAN WYK MA, VAN WYK BJ \& CHEN GA. 2009. A new hyperchaotic system and its circuit implementation. Chaos Solitons Fractals, 40: 2544-2549.

[4] VICENTE R, DAUDÉN J, COLET P \& TORAL R. 2005. Analysis and Characterization of the Hyperchaos Generated by a Semiconductor
Laser Subject to a Delayed Feedback Loop. IEEE J. Quantum Electron., 41: $541-548$.

[5] TAKAHASHI Y, NAKANO H \& SAITO T. 2004. A Simple Hyperchaos Generator Based on Impulsive Switching. IEEE Trans. Circuits Syst. II, 51: 468-472.

[6] BAIER G \& SAHLE S. 1994. Hyperchaos and chaotic hierarchy in low-dimensional chemical systems. J. Chem. Phys., 100: 8907-8911.

[7] JIA Q. 2007. Hyperchaos generated from the Lorenz chaotic system and its control. Phys. Lett. A, 366: 217-222.

[8] JIA Q. 2007. Adaptive control and synchronization of a new hyperchaotic system with unknown parameters. Phys. Lett. A, 362: 424-429.

[9] JIA Q. 2007. Projective synchronization of a new hyperchaotic Lorenz system. Phys. Lett. A, 370: 40-45.

[10] GRASSI G. 2009. Observer-based hyperchaos synchronization in cascaded discrete-time systems. Chaos Solitons Fractals, 40: 10291039.

[11] YASSEN MT. 2008. Synchronization hyperchaos of hyperchaotic systems. Chaos Solitons Fractals, 37: 465-475.

[12] WIGGINS S. 2003. Introduction to Applied Nonlinear Dynamical Systems and Chaos, Springer, New York.

[13] GUCKENHEIMER J \& HOLMES P. 2002. Nonlinear Oscillations. Dynamical Systems, and Bifurcations of Vector Fields, Springer, New York.

[14] SCHUSTER HG \& JUST W. 2005. Deterministic chaos. An introduction, WILEY-VCH, Weinheim. 